\title{
Multiple Mobile Robots Navigation and Obstacle Avoidance Using Minimum Rule Based ANFIS Network Controller in the Cluttered Environment
}

\author{
Anish Pandey* and Dayal R. Parhi \\ Mechanical Engineering Department, N.I.T, Rourkela, Orissa, India
}

Received: January 18, 2015; Accepted: January 27, 2016; Published: February 10, 2016

*Corresponding author: Anish Pandey, Mechanical Engineering Department, N.I.T, Rourkela, Orissa, India, E-mail: anish06353@gmail.com

\begin{abstract}
The motion control problem of an autonomous wheeled mobile robot has been widely investigated in the past two decades. In this article, the minimum rule based Adaptive Neuro-Fuzzy Inference System (ANFIS) controller has been presented for the safe navigation of single and multiple mobile robots in the cluttered environment by using the sensor-based steering angle control technique. The ultrasonic range finder sensor and sharp infrared range sensor have been used to read the front, right and left obstacle distance. This obstacle distance information is fed to the input of the ANFIS controller for selecting the suitable steering angle to achieve the collision-free path while mobile robots are moving to reach the goals in the cluttered environment. The advantages of this ANFIS network controller have a rapid response and can make the robots react efficiently in the real environment. The simulation result shows that the proposed ANFIS controller gives the safe, optimal and smooth travelling path in the cluttered environments. Moreover, the simulation results are compared with experimental results in the real environment to prove the authenticity the controller.
\end{abstract}

Keywords: Navigation; Sensors; Cluttered environment; Obstacle; Steering angle

\section{Introduction}

The present article explores the application of ANFIS network controller to solve the path planning problem of the multiple mobile robots to move from the current position to the goal position in the cluttered environment. The simulation and the experimental result show that the proposed ANFIS controller improves navigation performance in the unknown cluttered environments. ANFIS is a major topic in the field of neural and evolutionary soft computing. This algorithm plays a significant role in the designing of an intelligent autonomous control system. ANFIS makes the mapping between the input and output using the neural network and fuzzy logic for the better performance. This ANFIS architecture has been adapted from Matlab software package. ANFIS is a combination of fuzzy logic and Artificial Neural
Networks (ANN), where the fuzzy logic is capable of handling the linear and non-linear uncertainty, or both. The ANN is used to tune the parameters of the input and output membership functions [1]. ANFIS may be used as linear or non-linear, or both type dynamic systems, which solves the real system problem by using empirical dataset (experimental or predicted).

The ANFIS is the product of two methods, neural networks, and fuzzy systems. If both these intelligent methods are combined, better reasoning will be obtained in term of quality and quantity. In other words, both fuzzy reasoning and neural network calculation will be available simultaneously [7]. This ANFIS technique has been successfully applied by many researchers for sensor-based autonomous control mobile robots in the different environment. To address the best and optimal path, the robots required to move with suitable path planning algorithm, which calculates the minimum path length between any two points i.e. robot to obstacles or robot to the goal [3]. Pothal and Parhi [8] have proposed the sensor based Adaptive Neuro Fuzzy Inference System (ANFIS) controller for the navigation of single and multiple mobile robots in the highly cluttered environment. The authors have tried to design a control system architecture, which avoids the obstacle autonomously and reaches the target efficiently in all types of environments.

Hu and Brady [9] have described the design and implementation of parallel processing control architecture for sensor-based real-time local navigation of the mobile robots. The authors have done many experiments to prove the authenticity of the system. Contreras-Cruz, et al. [10] have proposed the evolutionary approach and artificial bee colony algorithm to solve the mobile robot path planning problem. Parhi and Mohanta [11] have developed the path planning of the multiple mobile robots in an unknown cluttered environment using a Petri-potential fuzzy hybrid controller with different membership functions. Furthermore, the set of sensors has attached to the robots to detect the nearby obstacles of the robot, to protect the robot from inter-collision. 
Mohanta, et al. [12] have integrated the Genetic Algorithm (GA) and Petri-Net technique for safe and collision-free path planning of the multiple robots with multiple targets in the cluttered environment. The Genetic Algorithm (GA) is used for finding an optimal or near optimal best paths in the cluttered and complex environments. Pradhan, et al. [13] have designed the navigation techniques for one thousand mobile robots using a fuzzy logic controller with different membership functions in an unknown environment.

$\mathrm{Hu}$, et al. [14] have designed the real-time path planning of a mobile robot by using the sensor-based neural network technique. The different sensors such as sonar sensors and infrared range finder sensors are used to control the steer of the robot to protect against unexpected hurdles in the uncertain terrain. Boubertakh, et al. [15] have prepared a new simple fuzzy logic eight rule-based controllers for mobile robot navigation, inspired by the human knowledge and used for obstacle avoidance and goal seeking behaviour. Kubota, et al. [16] have made the behaviour-based fuzzy controller for mobile robot navigation and collision avoidance in the non-stationary environment, and the conventional genetic algorithms (GAs) are integrated with for path optimization

Hoy, et al. [17] has studied the navigation problem of multiple vehicles through an unknown static environment with limited sensing and communication. Begum, et al. [18] has done the hybridization of fuzzy logic and genetic algorithm for solving the localization and mapping problem of several mobile robots. Korayem, et al. [18] have investigated the dynamic modelling and optimal point-to-point motion planning of a nonholonomic mobile robot in cluttered environments. Ahmadzadeh and Ghanavati [20] have proposed the PSO algorithm based navigation method for multiple mobile robots.

In this work, we propose the path planning and obstacle avoidance for multiple mobile robots in the cluttered environment using ANFIS network controller. Motivated by the aforementioned researchers, the primary objective of this article is to show how to guide the multiple autonomous mobile robots in an unknown cluttered environment using the minimum eight-rule based ANFIS controller. The proposed ANFIS network system is used to adjust the motions, directions and movements of the mobile robot to reach the goals with obstacle avoidance strategy. When the robots near to the obstacle, the obstacle avoidance behaviour a reactive, otherwise the goal finding behaviour will continue. The developed controller receives the input data Front Obstacle Distance (FOD), Right Obstacle Distance (ROD), and Left Obstacle Distance (LOD) from the various sensors. These sensor signals are fed to the input to the ANFIS network to provide the output Steering Angle (SA) control commands for the mobile robots. The proposed ANFIS controller trains the mobile robot system online dynamically. This ANFIS controller has three inputs and a single output. The input has two generalized bell-shaped (gbellmf) membership function, and output has Sugeno-type constant membership function. This paper is divided as follows. Section 1 presents the introduction. The dynamic modelling of the mobile robot is given in Section 2. ANFIS network architecture for multiple mobile robots navigation is proposed in Section 3. Section 4 shows the simulation result and discussion and also a comparison with previous works to the proposed method. Section 5 represents the experimental setup and its results and discussion for validating the proposed ANFIS controller. Finally, in Section 6, conclusions are presented.

\section{Dynamic Modeling of the Mobile Robot}

This work has been developed for wheeled type mobile robot, the front wheels of the robot are attached to the separate motors responsible for direction change (steer control), and the rear one is a caster wheel for balance. The movement of the mobile robot has been controlled by the steering (motor speed) of the front wheels. The dynamic modelling of the robot in the environment is shown in Figure 1. In Figure 1, $L, V_{R}$ and $V_{L}$ denotes the track width, right wheel velocity, and left wheel velocity of the mobile robot, respectively. The point $\mathrm{P}$ is located between the centres of the driving wheels axis. The landmark $(\mathrm{O}, \mathrm{X}, \mathrm{Y})$ is related to the field navigation environment. The central point $\left(X_{c^{\prime}} Y_{c}\right)$ is the middle of the two front steering wheels, and $\theta$ is the angle of steering about the axis $(\mathrm{O}, \mathrm{X})$. The following dynamic equation describes the motion and orientation of the robot in the field navigation environment: -

$$
\begin{aligned}
& X[j+1]=X[j]+V \cdot \cos \theta \\
& Y[j+1]=Y[j]+V \cdot \sin \theta \\
& \theta=\tan ^{-1}\left[\frac{G_{Y}-Y[j]}{G_{X}-X[j]}\right]
\end{aligned}
$$

Where $j=0,1,2, \ldots, \mathrm{n}$.

$$
V=\frac{\pi \times D \times N}{60} \mathrm{~m} / \mathrm{sec}
$$

Where $X[j]$ is the current position of the mobile robot in the $\mathrm{X}$-axis, and $\mathrm{X}[j+1]$ is the updated position of the robot when it moves forward by using Equation 1. Y [j] is the current position

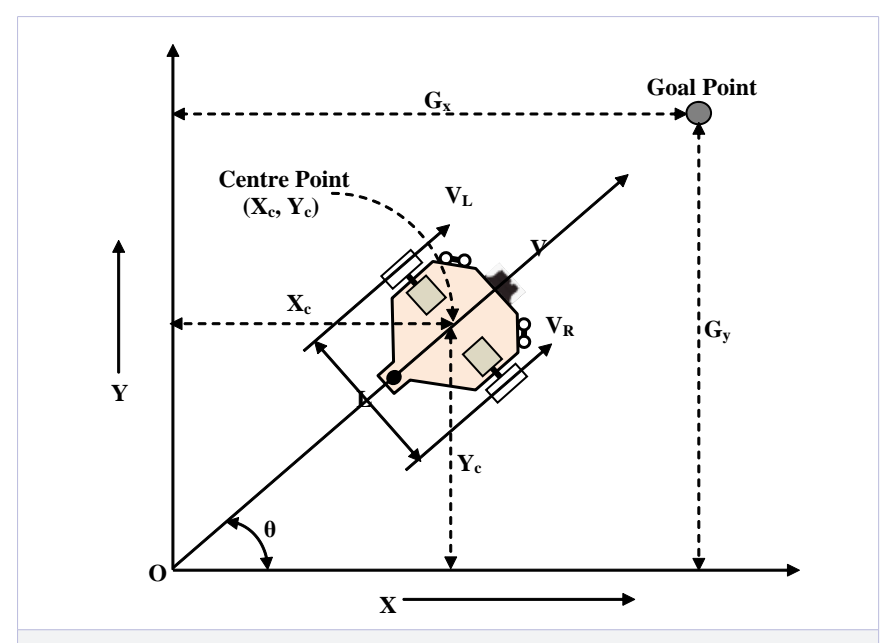

Figure 1: The dynamic modeling of a mobile robot. 
of the mobile robot in the Y-axis, and Y $[j+1]$ is the updated position of the robot when it moves forward by using Equation 2. The angle $\theta$ is the steering angle of the mobile robot, and this steering angle varies (or updated) according to the position of a goal in the environment. The $\mathrm{G}_{\mathrm{x}}$ and $\mathrm{G}_{\mathrm{y}}$ are the goal (or target) point in the $\mathrm{X}$ and $\mathrm{Y}$-axis, respectively. The $\mathrm{V}$ is the centre velocity in meter per second $(\mathrm{m} / \mathrm{sec})$, which depends upon the Diameter (D) of the wheel in meter, and the Angular Velocity (N) of the motor in RPM (Rounds Per Minute). The maximum and minimum velocity of the mobile robot used for navigation is $16.7 \mathrm{~cm} / \mathrm{sec}$, and $6.7 \mathrm{~cm} / \mathrm{sec}$ respectively.

The following conditions are used to control the motion and orientation (steering angle) of the mobile robot: -
If $V_{L}<V_{R}$, then the robot turns left side Figure 4

If $V_{L}>V_{R}$, then the robot turns right side Figure 5

If $V_{L}=V_{R}$, then the robot moves straight Figure. 6

\section{ANFIS Network Architecture For Multiple Mobile Robots Navigation}

\section{Controller Architecture}

The ANFIS controller for the multiple mobile robots navigation can be easily implemented for real-time applications because this controller handles unknown and uncertain situations with the simple computations. This navigation controller is working based on the local information collect from the different sensor.

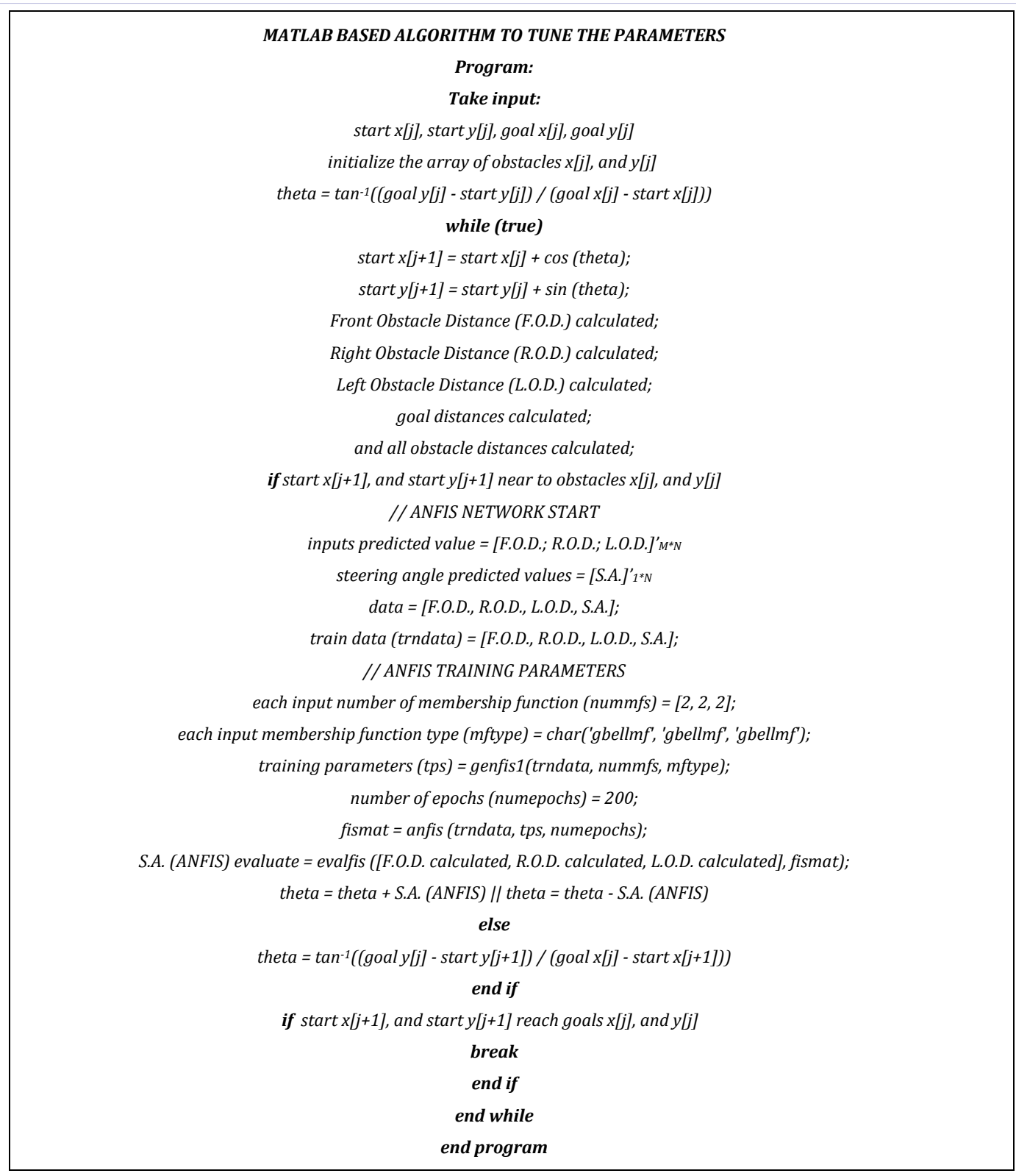

Figure 2: Matlab based algorithm to tune the parameters. 


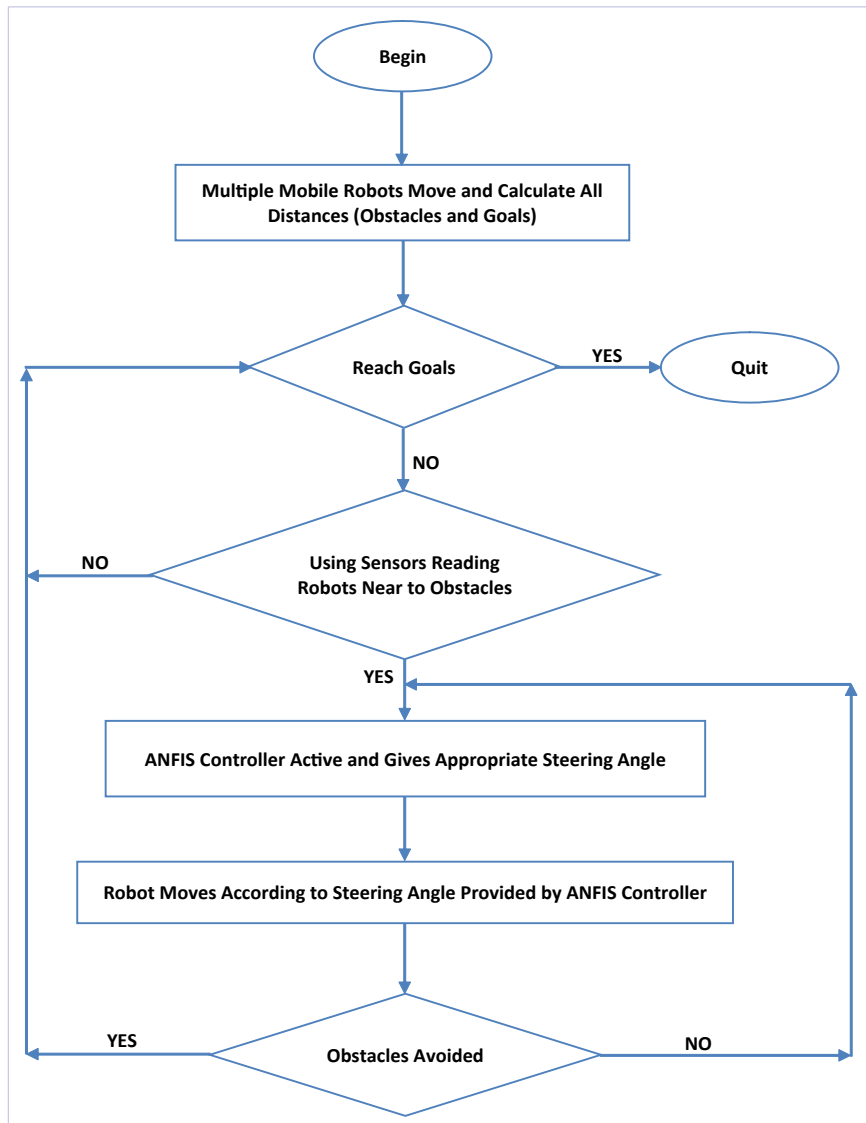

Figure 3: The architecture of mobile robots navigation based on ANFIS control system.

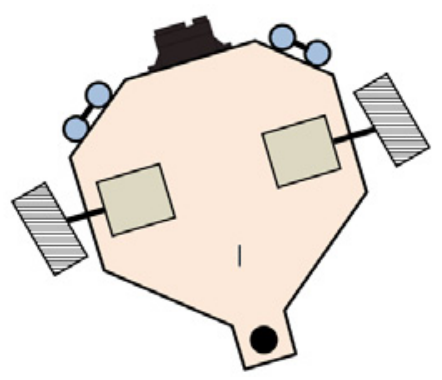

Figure 4: Positive steering angle.

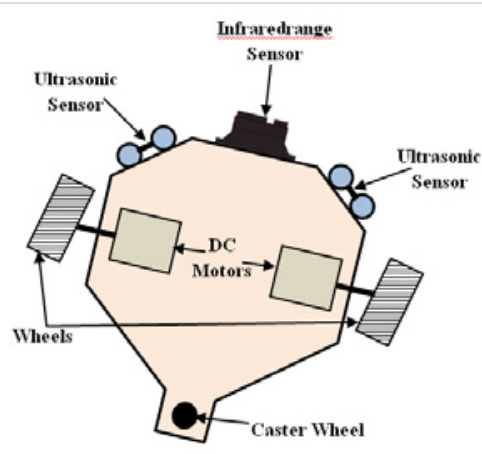

Figure 5: Negative steering angle.

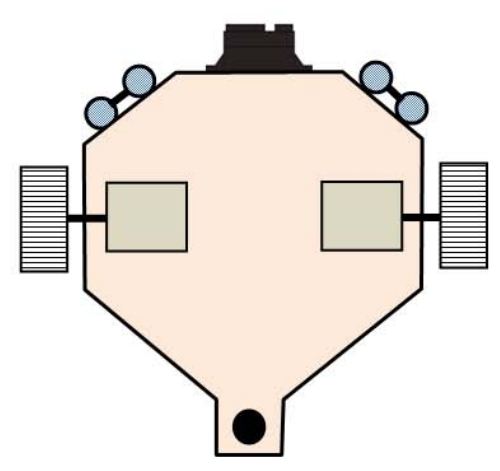

Figure 6: Zero steering angle.

For the safe navigation without any human intervention, the mobile robot should receive information about its surroundings environment by using the array of sensors. The proposed ANFIS controller has three inputs and a single output, the controller receives input (obstacle distance) from the ultrasonic range finder sensors and sharp infrared range sensor and provides the correct steering angle as an output. The training of this ANFIS model has been done by using fifteen data set given in Table 1 . The network is trained by fifteen specially designed sample data to deal the different behaviours. The ANFIS controller uses the set of sensors to detect the obstacle distance (front, right, and left) information as inputs and the steering angle as an output varies according to the obstacle distance data. Table 1 is described here, if the robots are finding obstacles at a distance $20 \mathrm{~cm}$ to the front, $20 \mathrm{~cm}$ to the right, and $100 \mathrm{~cm}$ to the left, then the mobile robot turns 74.3 degrees (left side or anticlockwise direction) means positive steering angle to reach the goals with obstacle avoidance Figure 4. If the robots are finding obstacles at a distance $20 \mathrm{~cm}$ to the front, $100 \mathrm{~cm}$ to the right, and $20 \mathrm{~cm}$ to the left, then the mobile robot turns-65.9 degrees (right side or clockwise direction) means negative steering angle to reach the goals in an environment with obstacle avoidance Figure 5. If the robot finds an obstacle at a distance $50 \mathrm{~cm}$ to the front, $15 \mathrm{~cm}$ to the right, and $15 \mathrm{~cm}$ to the left, then the mobile robot moves straight means zero degree steering angle to reach the goals in an environment with obstacle avoidance Figure 6 . If there are no obstacles near to the robot, goal finding behaviour becomes active. The negative steering angle means the right wheel velocity will be low, and the left wheel velocity will be high respectively, for ANFIS sensorbased control of multiple mobile robots navigations in the cluttered environment. The positive steering angle means the right wheel velocity will be increased much more than the velocity of the left wheel, for ANFIS sensor-based control of multiple mobile robot navigations in the cluttered environment. The zero steering angles means the both wheel velocities will move at the same speed, for ANFIS sensor-based control of multiple mobile robot navigations in the cluttered environment. The navigation algorithm plays a sensory act control cycle where the mobile robot senses the nearby obstacle to make the appropriate decisions in the every iteration. This ANFIS network controller collects the data from the array of sensors to control the speed of the robot according to the obstacle detection. Thus, the controller

Citation: Pandey A, Parhi DR (2016) Multiple Mobile Robots Navigation and Obstacle Avoidance Using Minimum Rule Based ANFIS Network Controller in the Cluttered Environment. Int J Adv Robot Automn 1(1): 1-11. http://dx.doi.org/10.15226/2473- 


\begin{tabular}{|c|c|c|c|c|c|}
\hline S. No. & $\begin{array}{l}\text { F.O.D. } \\
\text { 'cm' }\end{array}$ & $\begin{array}{l}\text { R.O.D. } \\
\text { 'cm' }\end{array}$ & $\begin{array}{l}\text { L.O.D. } \\
\text { 'cm' }\end{array}$ & \begin{tabular}{|l|} 
S.A. \\
'degree'
\end{tabular} & TURNING DIRECTION \\
\hline 1. & 20 & 20 & 100 & 74.3 & LEFT \\
\hline 2. & 20 & 100 & 20 & -65.9 & RIGHT \\
\hline 3. & 100 & 100 & 10 & -70.4 & RIGHT \\
\hline 4. & 25 & 50 & 75 & 55 & LEFT \\
\hline 5. & 40 & 60 & 100 & 59.4 & LEFT \\
\hline 6. & 15 & 100 & 100 & 72.8 & LEFT \\
\hline 7. & 25 & 100 & 50 & -22.9 & RIGHT \\
\hline 8. & 15 & 15 & 15 & 73.4 & LEFT \\
\hline 9. & 50 & 15 & 15 & 0 & STRAIGHT \\
\hline 10. & 20 & 10 & 10 & 77 & LEFT \\
\hline 11. & 100 & 15 & 15 & 0 & STRAIGHT \\
\hline 12. & 25 & 10 & 10 & 77.2 & LEFT \\
\hline 13. & 100 & 100 & 15 & -70.5 & RIGHT \\
\hline 14. & 100 & 25 & 20 & 0 & STRAIGHT \\
\hline 15. & 100 & 100 & 100 & -70.4 & RIGHT \\
\hline
\end{tabular}

provides the directional control of mobile robots, planning of its path to the reach goals, tracking a collision-free optimum travelling path in the environments. The controller has been designed by the Matlab ANFIS toolbox [4], and its programming is also done by the author. Their descriptions are shown in Figure 2 (Matlab based algorithm to tune the parameters). Figure 3 shows the architecture of our incorporated ANFIS control system to achieve the movement and direction (steering angle) control for the mobile robots.

\section{The Training Methodology}

ANFIS is a method of formulating the relationship between the input and the output using the fuzzy logic mathematical concept. There are the two types of Fuzzy Inference Systems (FIS) that can be classified as Mamdani-type and Sugeno-type. The ANFIS does work based on the Sugeno-type neuro-adaptive learning technique. This technique has more compact and computationally efficient, and its capability to customize the membership functions compare to the Mamdani-type fuzzy system. The adaptive techniques can be used to generate the membership function and rules automatically so that the fuzzy system delivers the best result from dataset [2]. In this paper, Adaptive NeuroFuzzy Inference System (ANFIS) is used, whose output is defined as the constant type. The final output of the ANFIS controller is calculated by the weighted average method. This proposed ANFIS controller has three input variables $x$ (F.O.D.), $y$ (R.O.D.), and $Z$ (L.O.D.), and a single output variable $f$ (S.A.), which is connected to each other by the fuzzy rules. The architecture of a typical ANFIS has three inputs, eight rules and a single output. The output is defined by the first-order function. The each input has two generalized bell-shaped (gbellmf) membership functions (MFs). Generally, the ANFIS controller works based on the firstorder Sugeno fuzzy model in the following form: -

$$
\begin{aligned}
& \text { Rule }_{i} \text { : If } x \text { is } M_{i}, y \text { is } N_{i}, \text { And } z \text { is } \\
& O_{i} \text { THEN } f_{i}=p_{i} \cdot x+q_{i} \cdot y+r_{i} \cdot z+s_{i}
\end{aligned}
$$

Where $i=1,2, \ldots, 8$, and the symbols $p_{i}, q_{i}, r_{i}$ and $S_{i}$ are the coefficients of output membership functions $f_{i}$ with the eight rules. $M_{i}, N_{i}$ and $O_{i}$ are the membership functions (MFs) of inputs $x, y$ and $z$ respectively of the fuzzy sets.

The Matlab software package is used to design the ANFIS architecture. The input layer addresses the Front Obstacle Distance (FOD), Right Obstacle Distance (ROD), and Left Obstacle Distance (LOD). The output layer addresses the Steering Angle (SA). In this architecture, the inputs are directly connected to the input membership functions (input mf); the input membership functions are connected to the rules; rules are connected to the output membership functions (output mf), and finally this output membership function is connected to the output. The input and output have three neurons and single neuron respectively. For training the ANFIS network, the TRAIN function of the Matlab software package is used. This function works on ANFIS hybrid learning algorithm, which is the combination of forward and backward neural network method. The proposed ANFIS architecture has five layers Figure 7, which perform the different actions for the controller. Their details are given below: -

Layer 1: The first layer is an adaptive node. It generates membership grade for the inputs. The outputs of this layer are defined as: -

$$
\begin{aligned}
& O_{M_{n}}^{1}=\mu_{M_{n}}(x) \\
& O_{N_{n}}^{1}=\mu_{N_{n}}(y) \\
& O_{O_{n}}^{1}=\mu_{O_{n}}(z)
\end{aligned}
$$

Citation: Pandey A, Parhi DR (2016) Multiple Mobile Robots Navigation and Obstacle Avoidance Using Minimum Rule Based ANFIS Network Controller in the Cluttered Environment. Int J Adv Robot Automn 1(1): 1-11. http://dx.doi.org/10.15226/2473- 


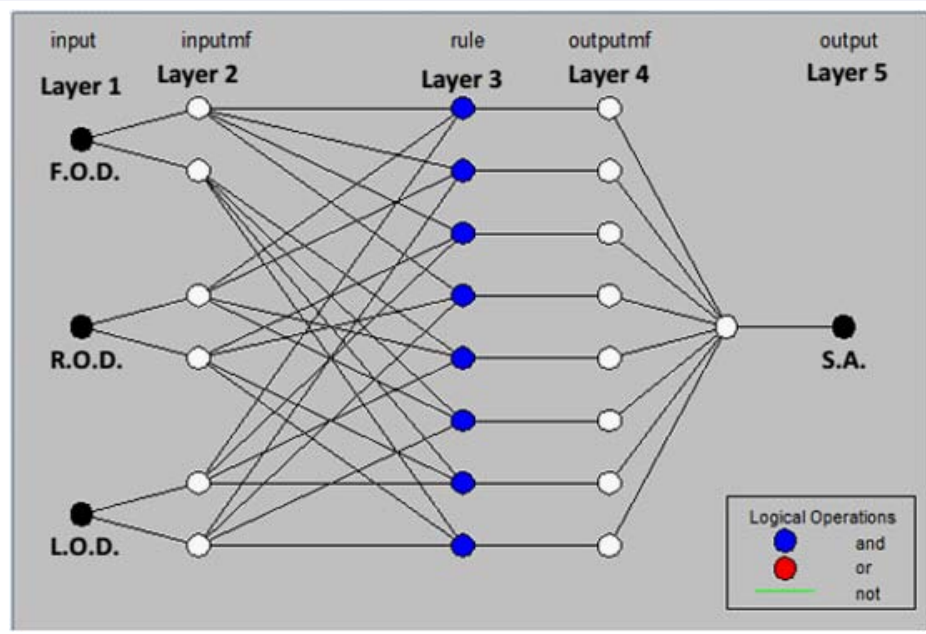

Figure 7: The general architecture of the ANFIS network.

Where $n=1,2, \ldots, 8$, and $\mu_{M_{n}}, \mu_{N_{n}}$ and $\mu_{O_{n}}$ are the input membership functions that can be triangular, trapezoidal, gaussian, generalized bell-shaped and other shape functions. In this paper, the following generalized bell-shaped membership function is used: -

$$
\mu_{M_{n}}(x ; a, b, c)=\frac{1}{1+\left|\frac{x-c}{a}\right|^{2 b}}
$$

The similar generalized bell-shaped membership equation will be written for $\mu_{N_{n}}$ and $\mu_{O_{n}}$ Where $a, b$ and $c$ are the premise parameters of the generalized membership function; called as the half width, slope control, and centre respectively.

Layer 2: The nodes in this layer are also called as the rule layer, indicating that they perform a simple multiplier. The outputs of this layer are represented as: -

$$
O_{n}^{2}=W_{n}=\mu_{M_{n}}(x) \cdot \mu_{N_{n}}(y) \cdot \mu_{O_{n}}(z)
$$

The $W_{n}$ represents a firing strength or the truth value, of the $\mathrm{n}^{\text {th }}$ rule and $n=1,2,3 \ldots 8$ is the number of Sugeno-type fuzzy rules.

Layer 3: It is also called as the normalization layer, which plays a normalization role in the ANFISnetwork. The outputs of this layer can be represented as: -

$$
O_{n}^{3}=\overline{W_{n}}=\frac{W_{n}}{\sum_{n=1}^{8} W_{n}}
$$

Layer 4: Every node in this layer is called adaptive node, whose output is simply the product of the normalized firing strength. A defuzzification node determines the weighted consequent value of a given rule. The connection between the inputs and output of this layer can be expressed as follows: -

$$
O_{n}^{4}=\overline{W_{n}} \cdot f_{n}=\overline{W_{n}}\left(p_{n} \cdot x+q_{n} \cdot y+r_{n} \cdot z+s_{n}\right)
$$

Layer 5: It is represented by a single summation of all incoming nodes. This single node is a fixed node, which determines the sum of the outputs of all defuzzification nodes and gives the overall system output that is steering angle as given below: -

$$
O_{n}^{5}=\sum_{n=1}^{8} \overline{W_{n}} \cdot f_{n}=\sum_{n=1}^{8}\left[\begin{array}{l}
\left(\overline{W_{n}} \cdot p_{n}\right) \cdot x+\left(\overline{W_{n}} \cdot q_{n}\right) \cdot y+ \\
\left(\overline{W_{n}} \cdot r_{n}\right) \cdot z+\overline{W_{n}} \cdot s_{n}
\end{array}\right]=\frac{\sum_{n=1}^{8} W_{n} \cdot f_{n}}{\sum_{n=1}^{8} W_{n}}
$$

The structure of ANFIS controller has two membership functions for all inputs, i.e. front, right and left obstacles. These inputs are specified in the form of generalized bell-shaped membership function. Because this generalized bell-shaped membership function covers a wide area, and, therefore, it delivers better results compared to other functions. The proposed ANFIS controller generates the rules and tunes the correct membership function grade from the dataset. During the learning process for the safe navigation of multiple mobile robots in the cluttered environment, the ANFIS modifies the inputs and output membership function parameters with the objective of minimizing the sum of square error of the output (Steering Angle). The training dataset is used to train the ANFIS network controller while the testing dataset is used to verify the accuracy and effectiveness of the trained ANFIS network controller for the computation of the data quality evaluation. The specifications of the proposed ANFIS network controller have been given in Table 2. The proposed ANFIS controller architecture is verified through the mean squared error (M.S.E.) method: -

$$
\text { M.S.E.(\%) }=\left[\sum_{1}^{k}\left(\frac{S A_{a}-S A_{p}}{k}\right)^{2}\right] \times 100
$$

Where $S A_{a}$ is the actual steering angle, $S A_{p}$ is the predicted steering angle value, and $k$ is the number of observations.

\section{Simulation Results and Discussion}

This section describes the successful simulation results using ANFIS controller in the various environments. Simulation result shows the method can be used for wheeled mobile robots moving 


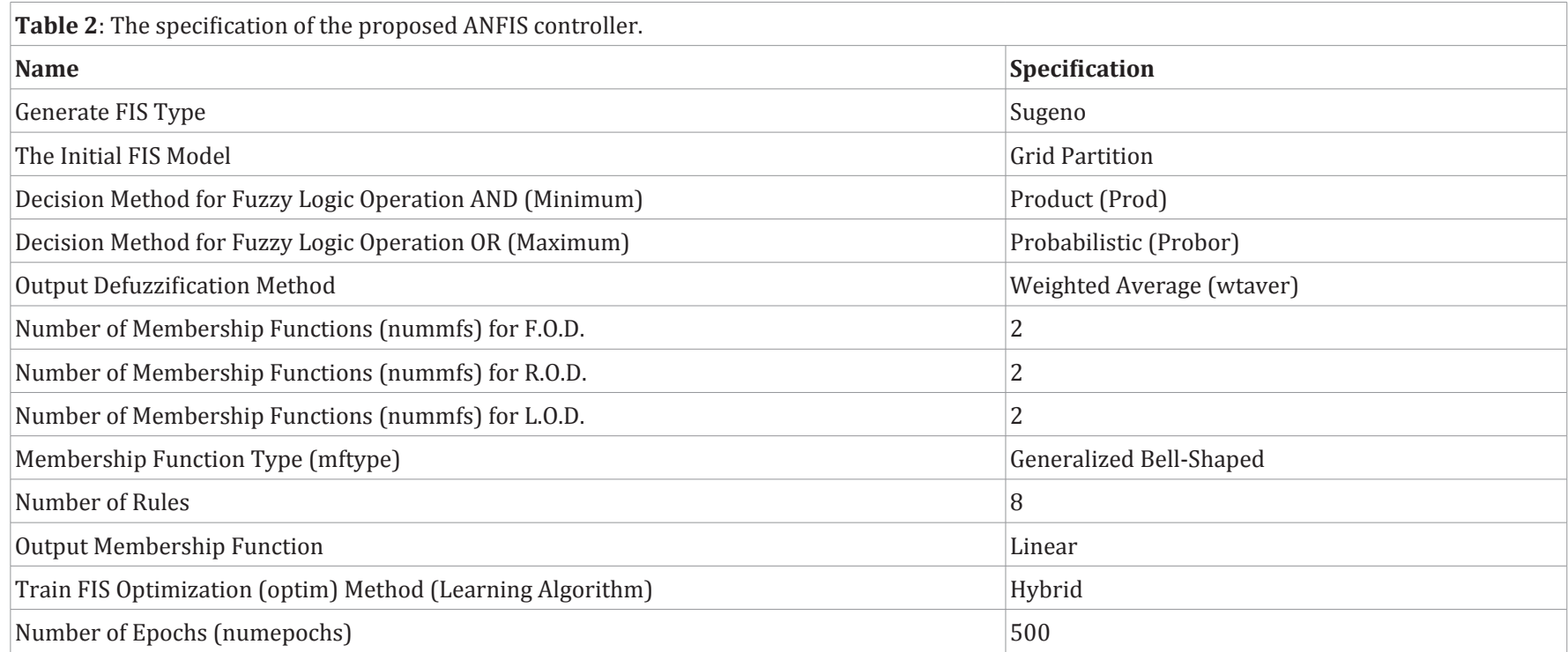

in the cluttered environment. The sensor data are used to control the mobile robots during simulation for the obstacle avoidance. Furthermore, the application of this ANFIS controller for mobile robots navigation has been also discussed. The simulation results in Figures 8-11 exhibit that the mobile robots start moving from start position avoid the many obstacles in the environment to reach goal positions with the possibility of minimum travelling path. The proposed navigation strategy is tested by simulating the three navigation actions such as obstacle avoidance, robots intercollision avoidance, and goal-seeking in the environment. The path traced by the single and multiple robots in the environment among the various obstacles using minimum rule based ANFIS network controller has been presented in Figures 8-12. The Figure 8 (i) and (ii) shows the navigation of single mobile robot in the different environments; Figure 9 shows the navigation of two mobile robots, Figure 10 shows the navigation of three mobile robots and Figure 11 shows the navigation of four mobile robots respectively. Total travelling path lengths and time taken to reach the goals in the both simulation and experiment modes are measured in centimetre by using statistical method for one, and two robot(s) respectively, and it is given in Table 3. Minimise the travelling path length and search time is the primary objectives for the mobile robots navigation in different environments. From the simulation results, it is clear that, the developed ANFIS controller can drive the robots smoothly without collision in the cluttered environment. Moreover, the experimental results of these simulations have been shown in Figures 16-17

\section{Comparison with Previous Works}

This section describes the simulation result comparisons between the Zhang, et al. [5], Montaner, et al. [6] models and the proposed minimum rule based ANFIS network controller. The performance of this ANFIS controller is evaluated on the basis of the following criteria:-

(a) Travelling path length

(b) Travelling path smoothness

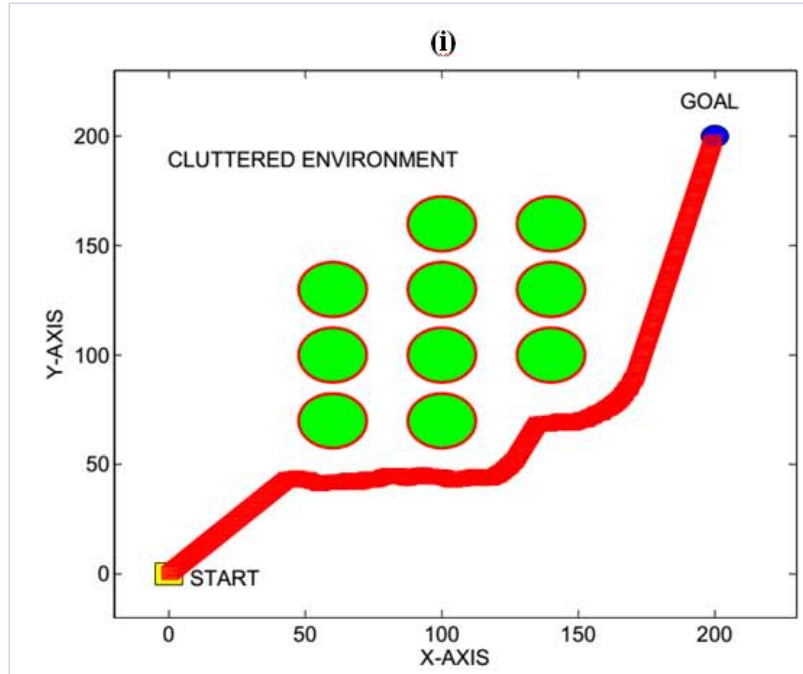

(ii)

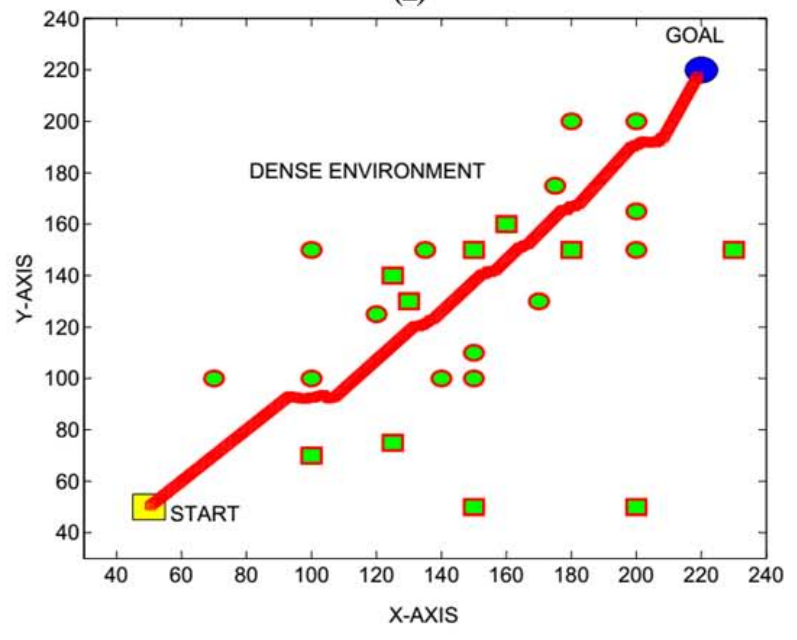

Figure 8: Obstacle avoidance in the different cluttered environment by the single mobile robot using minimum rule. 


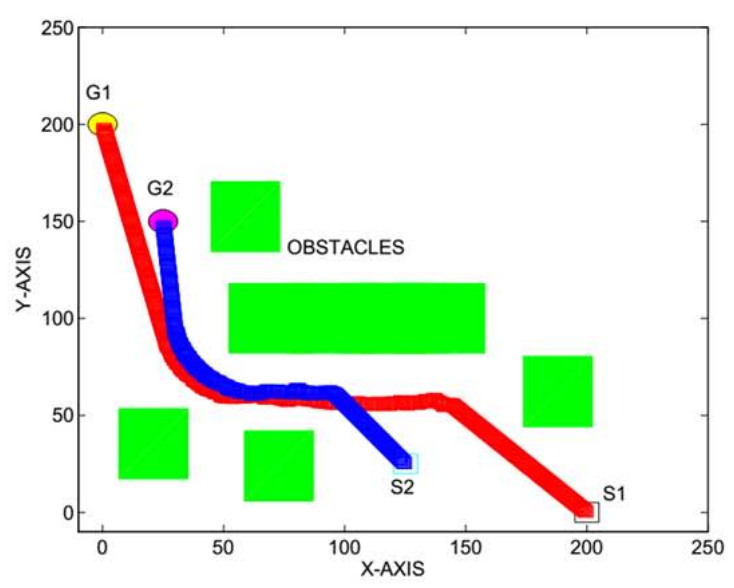

Figure 9: Obstacle avoidance in the environment by two mobile robots with two goals using the minimum rule based ANFIS network controller.

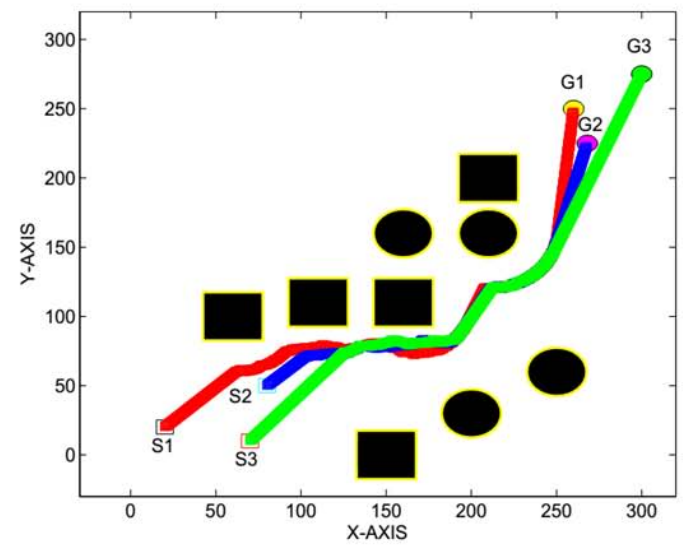

Figure 10: Obstacle avoidance in the environment by three mobile robots with three goals using the minimum rule based ANFIS network controller.

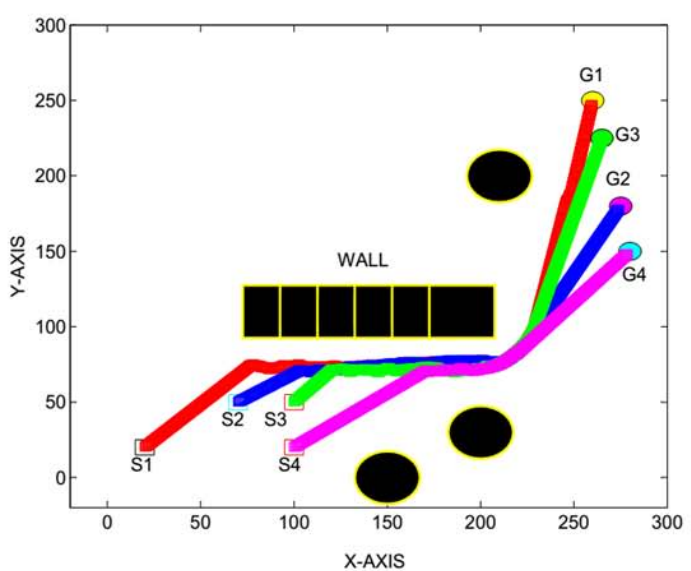

Figure 11: Obstacle avoidance in the environment by four mobile robots with four goals using the minimum rule based ANFIS network controller.
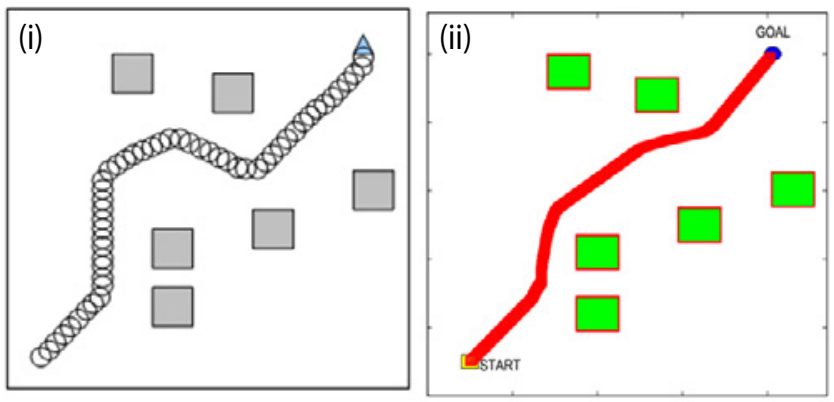

Figure 12: The simulation results comparison between the (i) Zhang et al. [5] model and the (ii) ANFIS controller in the same environment.

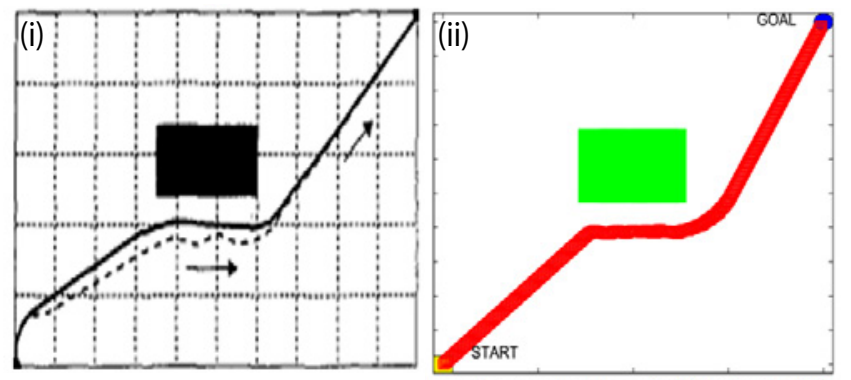

Figure 13: The simulation results comparison between the (i) Montaner et al. [6] model and the (ii) ANFIS controller in the same environment.
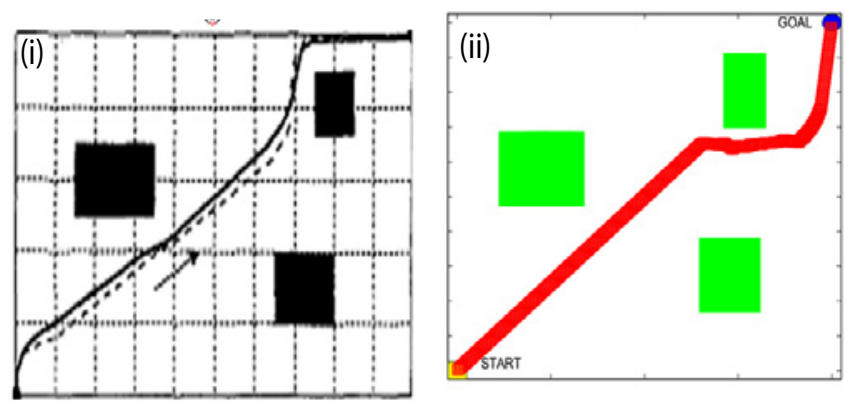

Figure 14: The simulation results comparison between the (i) Montaner et al. [6] model and the (ii) ANFIS controller in the same environment.

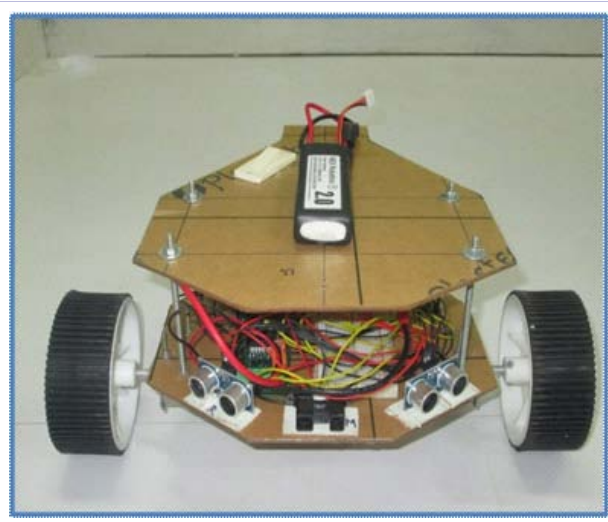

Figure 15: Experimental mobile robot.

Citation: Pandey A, Parhi DR (2016) Multiple Mobile Robots Navigation and Obstacle Avoidance Using Minimum Rule Based ANFIS Network Controller in the Cluttered Environment. Int J Adv Robot Automn 1(1): 1-11. http://dx.doi.org/10.15226/2473- 
Zhang, et al. [5] have developed the reactive fuzzy logic based control strategy for mobile robot navigation. The authors have integrated this fuzzy logic controller with the RAM based neural network to decrease the number of fuzzy rules. Where the fuzzy rule-based controller are used to interpret sensory information and the neural network provides the appropriate actions to avoid obstacles and does control the heading angle of the robot. The four sharp infrared (IR) range sensors are mounted on the left, right, left-front and right-front side of the mobile robot to detect the obstacles. The two stepper motors are used to drive the each rear wheel to facilitate turns or forward and backward movement.

Montaner, et al. [6] have designed the fuzzy logic controller for autonomous mobile robot path planning, which reduces the navigation time from a start position to the end position in an environment. They developed a fuzzy controller based experimental mobile robot that establish a map between input space (information receiving from ultrasonic sensors) to control the direction and velocity (output space) in real time autonomously.

The performance of this proposed ANFIS controller is mainly evaluated by navigation path length and path smoothness. Figures 12-14 show the simulation comparisons between the ANFIS controller and the previous models [5-6]. Table 4 and 5 show the path traced by the robot using the ANFIS controller and the previous models [5-6] in the various environments. In Table

Table 3: Navigation path lengths by the robots during simulation and experiment.

\begin{tabular}{|l|l|l|l|l|}
\hline \multirow{2}{*}{ Figures description } & \multicolumn{2}{l|}{ Navigation path length in 'cm' } & \multicolumn{2}{l}{ Time taken to reach the goals in 'second' } \\
\cline { 2 - 5 } & Simulation result & Experimental result & Simulation result & Experimental result \\
\hline Fig.8 (i) and Fig.16 Single mobile robot & 107.2 & 115.3 & 9.4 & 10.2 \\
\hline Fig.9 and Fig.17 Two mobile robots & 103.5 and 57.1 res. & 111.7 and 61.8 res. & 9.2 and 5.1res. \\
\hline
\end{tabular}

Table 4: The Results of path traced by the robot using the ANFIS controller and the Zhang et al. [5] model.

\begin{tabular}{|l|l|l|l|}
\hline Fig. no & Number of robots & $\begin{array}{l}\text { Path traced by the robot using Zhang et al. [5] } \\
\text { model in 'cm' }\end{array}$ & $\begin{array}{l}\text { Path traced by the robot using ANFIS controller } \\
\text { in 'cm' }\end{array}$ \\
\hline Fig.12 (i), (ii) & 1 & 91.3 & 78.9 \\
\hline
\end{tabular}

Table 5: The Results of path traced by the robot using the ANFIS controller and the Montaner et al. [6] model.

\begin{tabular}{|c|c|c|c|}
\hline Fig. no & Number of robot & $\begin{array}{l}\text { Path traced by the robot using Montaner et al. [6] } \\
\text { model in 'cm' }\end{array}$ & $\begin{array}{l}\text { Path traced by the robot using ANFIS controller } \\
\text { in 'cm' }\end{array}$ \\
\hline Fig.13 (i), (ii) & 1 & 100.7 & 95.2 \\
\hline
\end{tabular}

Table 6: Experimental mobile robot specifications.

\begin{tabular}{|c|c|}
\hline Name & Specification \\
\hline Microcontroller & Arduino UNO ATmega328 \\
\hline Flash Memory & 32 KB (ATmega328) \\
\hline Operating Voltage & $5 \mathrm{~V}$ \\
\hline SRAM & 2 KB (ATmega328) \\
\hline InputVoltage (Recommended) & $7-12 \mathrm{~V}$ \\
\hline Input Voltage (Limits) & $6-20 \mathrm{~V}$ \\
\hline Analog Input Pins & 6 \\
\hline Motors & 2 DC, 30RPM Centre Shaft Economy Series DC Motor \\
\hline Motors Driver & L298, Up to 46V, 2A Dual DC Motor Driver \\
\hline Motor Speed & Max: 30RPM, Min: 12RPM \\
\hline Wheel & Wheel Diameter: $106 \mathrm{~mm}$, Wheel Thickness: $44 \mathrm{~mm}$, Hole Diameter: $8 \mathrm{~mm}$ \\
\hline Sensors & 1 IR Range Sensor Distance Measuring Range: $20 \mathrm{~cm}$ to $150 \mathrm{~cm}$ \\
\hline Bread Board & Small Size Bread Board \\
\hline Communication & USB connection Serial Port \\
\hline Size & Height: $7.5 \mathrm{~cm}$, Length: $27 \mathrm{~cm}$, Width: $33 \mathrm{~cm}$, \\
\hline Weight & Approx. $1.4 \mathrm{~kg}$ \\
\hline Payload & Approx. 400g \\
\hline Power & Rechargeable Lithium Polymer 3 Cell, 11.1V, 2000mAh, 20C Battery \\
\hline
\end{tabular}

Citation: Pandey A, Parhi DR (2016) Multiple Mobile Robots Navigation and Obstacle Avoidance Using Minimum Rule Based ANFIS Network Controller in the Cluttered Environment. Int J Adv Robot Automn 1(1): 1-11. http://dx.doi.org/10.15226/2473- 
4, the path traced by the robot has been found $91.3 \mathrm{~cm}$ and $78.9 \mathrm{~cm}$ by using Zhang, et al. [5] model and the current ANFIS controller, respectively in the same environment. Similarly, in Table 5 , the path covered by the robot has been found $100.7 \mathrm{~cm}$ and $95.2 \mathrm{~cm}$ and so on, by applying Montaner, et al. [6] model and current ANFIS controller, respectively in the same environment. From the simulation comparisons, it is clearly seen that, the developed ANFIS controller efficiently drives the robots in the same path planning problem.

\section{Experimental Results and Discussion}

The proposed ANFIS controller has implemented on the real experimental mobile robot Figure 15. The robot has two front driving wheels attached to the separate motors used for Steering Angle (SA) and driving (motor speed) control and single caster wheel for balance the robot. Two separate DC motors are used to drive each front wheel to facilitate turns, backward and forward movement. The speed of the motors is controlled by the dual DC motor driver L298, which is connected to the Arduino UNO (ATmega328) microcontroller. If there are no obstacles detected nearby the robot, then the right and left motors will move at the same speed; otherwise, it will slow down. If there are obstacles, detect right side, then the robot turns positive steering angle means the right and left motors will move high and low speed respectively. If there are obstacles, detect left side, then the robot turns negative steering angle means right and left motors will move low and high speed respectively. The mobile robot is equipped with a single sharp Infrared (IR) Range sensor for close obstacle detection, and two ultrasonic range finder sensors are used for the purpose of sensing obstacles around the robot. The sensors are evenly mounted on the front, right and left side of the robot. Ultrasonic range finder sensors are equipped on the left, and right side of the mobile robot, which measures distance between $2 \mathrm{~cm}$ to $400 \mathrm{~cm}$ and single sharp Infrared (IR) Range sensor are attached on the front of the mobile robot, which reads the distance between $20 \mathrm{~cm}$ to $150 \mathrm{~cm}$. The other specifications of the experimental mobile robot are given in Table 6.

To demonstrate the effectiveness of an ANFIS network controller a variety of experiments have been done with the real experimental mobile robot Figure 15. The resulting algorithm has been burnt on the $\mathrm{C} / \mathrm{C}++$ running microcontroller based experimental mobile robot and tested in the various cluttered environments for the comparison of the performances. The experimental verification of the above simulation results has been shown in Figure 16 and Figure 17. In Figure 16, the single robot has been used for the navigation. Similarly, in Figure 17, multiple robots have been used for the navigation. The dimension of the environment is $300 \mathrm{~cm}$ width and $300 \mathrm{~cm}$ height. We have set a threshold distance between the robots and obstacles, if the robot detects the obstacle in the threshold range, then the proposed ANFIS controller is active, and the robot moves/turns according to the ANFIS controller output (steering angle). From the above simulation and experimental results, it can be clearly seen that the ANFIS network controller efficiently drives the robot safely in the different cluttered environment. The navigation path length result between the simulation and experimental is listed in Table 3.

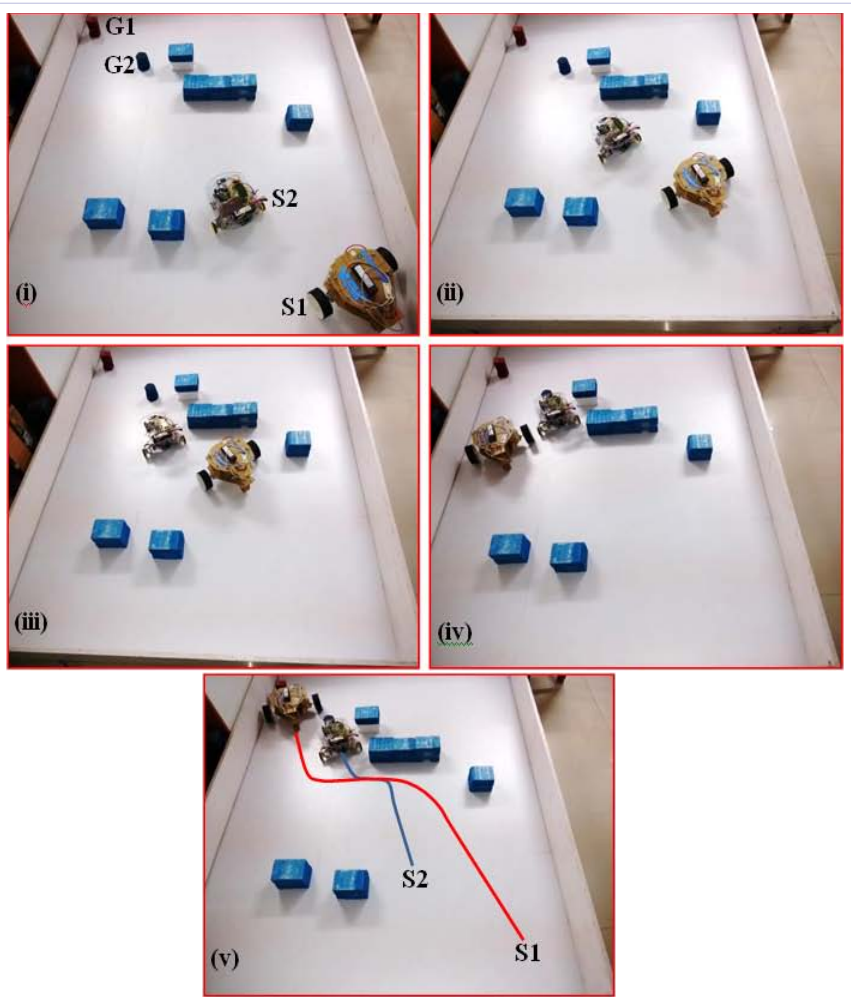

Figure 16: Experimental results for the navigation of mobile robot same as a simulation environment shown in Figure 8 (i).

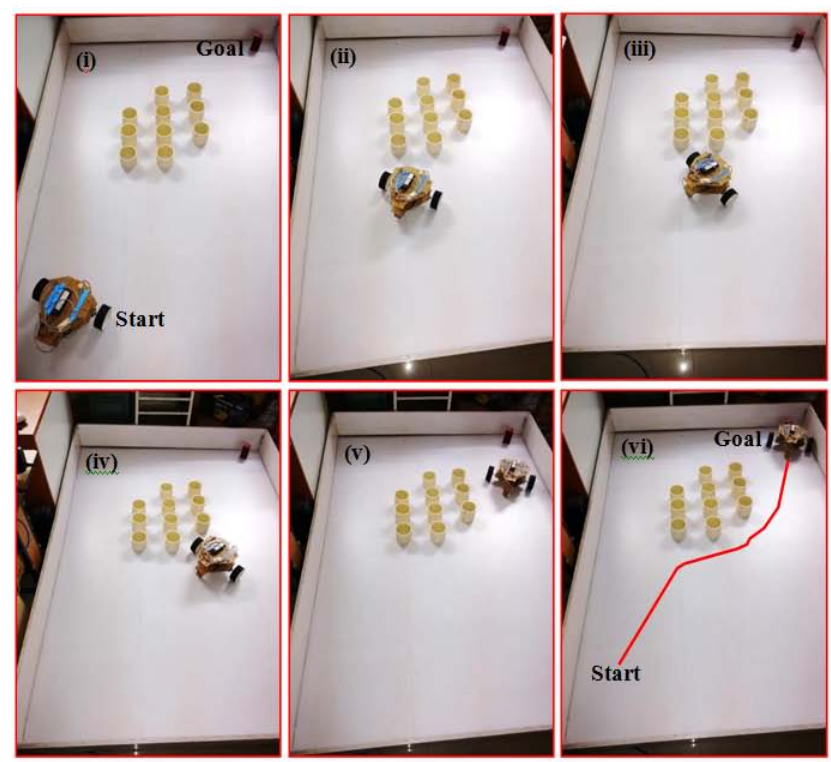

Figure 17: Experimental results for the navigation of two mobile robots same as a simulation environment shown in Figure 9.

\section{Conclusion}

In the current study, we have described the application of the ANFIS network controller for multiple mobile robots navigation and the obstacle avoidance in the cluttered environments. The ANFIS controller has three inputs and a single output. The 
controller receives inputs (obstacle distances) from different sensors to provide appropriate Steering Angle (SA) control command as output. Under the supervision of the proposed ANFIS controller, the mobile robots are autonomously reaching the goals with optimal and smooth travelling path. The eight rules and different behaviors such as obstacle avoidance, robots inter-collision avoidance, and the goal seeking behaviour has been used for the navigation of multiple mobile robots, which is less than the other conventional approaches i.e. forty-seven rules made by Zhang et al. [5] and forty-five rules made by Montaner, et al [6]. In addition, the effectiveness and efficiency of the ANFIS network controller have been verified through various simulations and experiments in the real environment. In future work, this ANFIS controller may be implemented in the dynamic environment with the hybridization of other nature-inspired algorithms for multiple dynamic obstacles and dynamic goal path planning problems.

\section{References}

1. Mucsi K, Ata MK, Ahmadi M. An Adaptive Neuro-Fuzzy Inference System for Estimating the Number of Vehicles for Queue Management at Signalized Intersections. Transportation Research. 2011;19(6):1033-1047.

2. Hamidian D, Seyedpoor SM. Shape Optimal Design of Arch Dams Using an Adaptive Neuro-Fuzzy Inference System and Improved Particle Swarm Optimization. Applied Mathematical Modelling. 2010;34(6):1574-1585.

3. Jaradat MA, Mohammad R, Quadan L. Reinforcement Based Mobile Robot Navigation in Dynamic Environment. ELSEVIER Robotics and Computer-Integrated Manufacturing. 2011;27(1):135-149.

4. Matlab 2012 a. ANFIS Toolbox

5. Zhang N, Beetner D, Wunsch DC, Hemmelman B, Hasan A. An Embedded Real-Time Neuro-Fuzzy Controller for Mobile Robot Navigation. IEEE International Conference on Fuzzy Systems. 2005;pp.319-324.

6. Montaner MB, Ramirez SA. Fuzzy Knowledge-Based Controller Design for Autonomous Robot Navigation. Expert Systems with Applications. 1998;4(1):179-186.

7. Imen M, Mohammad M, Shoorehdeli MA. Tracking Control of Mobile Robot Using ANFIS. IEEE International Conference on Mechatronics and Automation. 2011;pp.422-427.

8. Pothal JK, Parhi DR. Navigation of Multiple Mobile Robots in a Highly Clutter Terrains using Adaptive Neuro-Fuzzy Inference System.
Robotics and Autonomous Systems. 2015;72:pp.48-58. doi:http:// dx.doi.org/10.1016/j.robot.2015.04.007.

9. Hu H, Brady M. A Parallel Processing Architecture for Sensor-Based Control of Intelligent Mobile Robots. Robotics and Autonomous Systems. 1996;17(4):235-257.

10. Contreras-Cruz MA, Ayala-Ramirez V, Hernandez-Belmonte UH. Mobile Robot Path Planning Using Artificial Bee Colony and Evolutionary Programming. Applied Soft Computing. 2015;30:319328.

11. Parhi DR, Mohanta JC. Navigational Control of Several Mobile Robotic Agents Using Petri-Potential-Fuzzy Hybrid Controller. Applied Soft Computing. 2011;11(4):3546-3557.

12. Mohanta JC, Parhi DR, Patel SK. Path Planning Strategy for Autonomous Mobile Robot Navigation Using Petri-GA Optimisation. Computers and Electrical Engineering. 2011;37(6):1058-1070.

13. Pradhan SK, Parhi DR, Panda AK. Fuzzy Logic Techniques for Navigation of Several Mobile Robots. Applied Soft Computing. 2009;9(1):290-304.

14. Hu E, Yang SX, Chiu DK, Smith WR. Real-Time Tracking Control with obstacle avoidance of multiple mobile Robots. IEEE Proceedings of the International Symposium on Intelligent Control. 2002;pp.87-92. doi:10.1109/ISIC.2002.1157743.

15. Boubertakh H, Tadjine M, Glorennec P, Labiod S. A Simple Goal Seeking Navigation Method for a Mobile Robot using Human Sense, Fuzzy Logic and Reinforcement Learning. Journal of Automatic Control, Journal of Automatic Control. 2008;18(1):23-27.

16. Kubota N, Morioka T, Kojima F, Fukuda T. Learning of Mobile Robots Using Perception-Based Genetic Algorithm. Measurement. 2001;29(3):237-248.

17. Hoy M, Matveev AS, Savkin AV. Collision Free Cooperative Navigation of Multiple Wheeled Robots in Unknown Cluttered Environments. Robotics and Autonomous Systems. 2012;60(10):1253-1266.

18. Begum M, Mann GK, Gosine RG. Integrated Fuzzy Logic and Genetic Algorithmic Approach for Simultaneous Localization and Mapping of Mobile Robots. Applied Soft Computing. 2008;8(1):150-165.

19. Korayem M. H, Nazemizadeh M, Nohooji HR. Optimal Point-to-Point Motion Planning of Non-Holonomic Mobile Robots in the Presence of Multiple Obstacles. Journal of the Brazilian Society of Mechanical Sciences and Engineering2014;36(1):212-232.

20. Ahmadzadeh S, Ghanavati M. Navigation of Mobile Robot Using the PSO Particle Swarm Optimization. Journal of Academic and Applied Studies(JAAS). 2012;2(1):32-38. 